\title{
Direct immunofluorescence of skin using formalin-fixed paraffin-embedded sections
}

\author{
SL MERA, EW YOUNG, AND JWB BRADFIELD \\ From the University Department of Pathology, University Walk and Bristol Royal Infirmary, Bristol, \\ Avon, UK
}

SUMMARY The technique of direct immunofluorescence has been applied to skin biopsy specimens fixed in formalin and embedded in paraffin wax. The results have been compared with those obtained by using snap-frozen biopsy specimens from the same patients. Trypsinisation of the dewaxed material allowed subsequent detection of immunoglobulins, complement, and fibrinogen. When compared to the fluorescence in the snap-frozen specimens, the staining in the paraffin sections was less bright and there was a higher rate of negatives. Even so, it was possible to establish the diagnosis in most cases of pemphigus, pemphigoid, and lupus erythematosus.

The technique of direct immunofluorescence to demonstrate immunoglobulins, complement, and fibrinogen is of proven value in the diagnosis of several different skin conditions. ${ }^{12}$ One of the major disadvantages of this procedure is the requirement for fresh frozen tissue. Problems arise because (1) facilities for the snap-freezing of fresh specimens are not always available in all clinical areas, (2) the transportation of frozen tissue may be difficult, especially between hospitals, and the use of special holding fixatives to allow transportation before freezing may lead to loss of antigen reactivity, ${ }^{3}$ (3) the long-term storage of frozen tissue also leads to loss of antigen reactivity. ${ }^{4}$ Finally, formalin-fixed material is sometimes the only tissue available either because the lesion was too small to divide or because stored material is being reviewed retrospectively. All of these disadvantages would be avoided if formalin-fixed paraffin-embedded tissues could be used for direct immunofluorescence. We describe here the results of a study which was done to assess the possibility of using such material in a diagnostic skin immunofluorescence service.

\section{Material and methods}

SELECTION OF BIOPSY MATERIAL

Six cases each of pemphigus, pemphigoid, and lupus erythematosus were studied. The diagnosis was established using clinical features, conventional light microscopy, and direct immunofluorescence of

Received for publication 4 October 1979 fresh frozen tissue. The six control biopsies were from patients with uncelated skin disorders which showed minimal histological abnormalities and entirely negative direct immunofluorescence on frozen tissue.

PREPARATION OF BIOPSY TISSUE

Two $5 \mathrm{~mm}$ punch biopsies were obtained from each patient.

\section{Cryostat sections}

One of the two specimens was immediately snapfrozen in liquid nitrogen. After storage in liquid nitrogen for not longer than seven days the frozen tissue was mounted in OCT compound (Lab-Tek, Ames) and sectioned at $6 \mu$ in a cryostat at $-20^{\circ} \mathrm{C}$. The sections were then stained by the direct immunofluorescence procedure without prior fixation or trypsinisation.

\section{Paraffin sections}

The other punch biopsy was fixed in $10 \%$ nonbuffered formol saline, processed, and embedded in paraffin wax using standard laboratory procedures. Five micron wax sections were cut. For histological staining, these were dewaxed and stained with haematoxylin and eosin. For immunofluorescence, sections were dried overnight at $37^{\circ} \mathrm{C}$.

TR Y PSINISATION

Before immunofluorescent staining, dewaxed and rehydrated sections were rinsed in Tris-saline buffer, $\mathrm{pH} 7 \cdot 8$, and then trypsinised at $37^{\circ} \mathrm{C}$ in a solution of 
Tris-saline buffer containing $\mathbf{0} \cdot 1 \%$ calcium chloride and $0.05 \%$ trypsin (Wellcome). After a series of preliminary investigations to document the effects of varying the times of trypsinisation (see Results section) a standard incubation time of $\mathbf{4 0}$ minutes was adopted. After trypsin treatment the sections were rinsed and left overnight at $4{ }^{\circ} \mathrm{C}$ in Tris-saline buffer.

\section{IMMUNOFLUORESCENT STAINING}

Both frozen and trypsinised formalin-fixed sections were treated similarly. Before staining they were rinsed with two changes of phosphate buffered saline (PBS), $\mathrm{pH} 7 \cdot 3$. They were then incubated in a moist chamber for $\mathbf{3 0}$ minutes at room temperature with commercially available fluorescein conjugates of rabbit-antihuman $\operatorname{IgG}, \operatorname{IgM}, \operatorname{IgA}, \mathrm{C} 3$, and fibrinogen (Dakopatts, Mercia Brocades). After staining they were washed in three changes of PBS before being mounted in PBS/glycerol.

The effect of variations in the titres of antisera were initially investigated using serial dilutions ranging from neat antisera to $1 / 320$. There was optimal staining when the immunofluorescence was at a maximum compared with the background fluorescence; this occurred at a dilution of $1 / 10$ for each of the conjugated antisera in both frozen and paraffin sections. It was not possible to raise the intensity of fluorescence in the fixed material by increasing the concentration of the reagents to more than $1 / 10$ without causing an unacceptable level of background fluorescence. A dilution of $1 / 10$ was therefore used for the staining of both frozen and paraffin sections.

\section{SPECIFICITY}

The purpose of this study was to compare the patterns of skin immunofluorescence in frozen and formalin-fixed paraffin-embedded sections from the same patients. Because of this the possibility of variation in staining patterns between individual batches of antisera was excluded not by specific blocking procedures but by the incorporation of known positive and negative controls. In addition, all the paraffin-embedded material was stained on at least two separate occasions with different batches of antisera.

EXAMINATION OF TREATED SECTIONS All the preparations were examined independently by at least two investigators using a Zeiss Universal microscope equipped with FITC excitor and barrier filters and incident illumination from an Osram HBO 50 lamp. Staining patterns were noted and the intensity of fluorescence was graded from 0 to $+++$

\section{Results}

GENERAL

In the formalin-fixed paraffin-embedded material IgG, IgM, IgA, C3, and fibrinogen were all detected. Trypsin pretreatment was essential. A preliminary study showed that when trypsin incubation times of less than 20 minutes were used the numbers and the intensity of positive results were both reduced. In contrast, treatment for more than 1 hour led not only to poorer discrimination between specific immunofluorescence and background staining but also to an unacceptably high loss of sections from the slides.

The patterns of immunofluorescence were identical in both fixed and frozen material, although the intensity of fluorescence was usually less in the sections of fixed tissue. In the control groups, selected because of entirely negative direct immunofluorescence on frozen section, there were no positive results using formalin-fixed paraffinembedded sections.

\section{PEMPHIGUS}

Within the epidermis intercellular staining for IgG could be demonstrated in most of the formalin-fixed paraffin-embedded sections studied, and its pattern (Fig. 1) exactly resembled that seen in frozen material. Table 1 shows that, of the six cases selected for their intercellular staining for IgG on frozen section, five were positive for IgG using paraffin-embedded material, although the intensity of fluorescence was usually reduced. The remaining one negative case remained so on repeat study. The cases that were positive for IgM and IgA on frozen section were all negative on the paraffin-embedded material. The five cases that on frozen section showed positive staining for $\mathrm{C} 3$, and the three that were positive for fibrinogen, were all negative using paraffin-embedded sections. None of the paraffin sections gave positive immunofluorescence unless the frozen sections had also been positive.

PEMPHIGOID

Immunofluorescent staining for IgG at the basement membrane zone could be demonstrated in all of the formalin-fixed paraffin-embedded sections studied, and its pattern, shown in Fig. 2, exactly resembled that seen in the frozen material. Table 2 shows that, of the six cases selected for their staining for IgG at the basement membrane zone in frozen sections, all six showed similar staining using paraffin-embedded sections. A similar pattern of staining for $\mathrm{C} 3$ was seen in paraffin-embedded sections in four out of the five cases that were positive in the frozen sections. In most cases the 


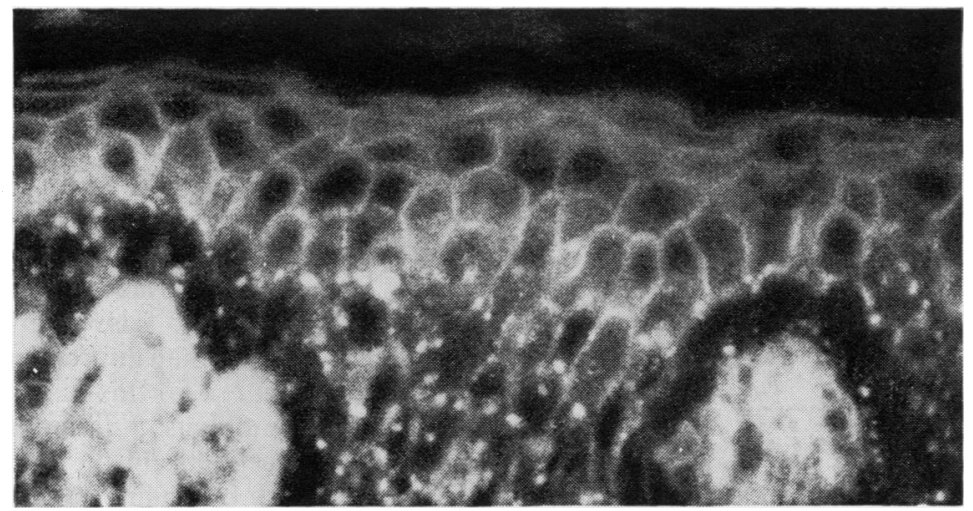

Fig. 1 Pemphigus: intercellular IgG. Paraffin section, direct immunofluorescence $\times 500$

Table 1 Pemphigus: intercellular staining

\begin{tabular}{lllllll}
\hline Case & Tissue & IgG & IgM & IgA & C3 & Fibrin \\
\hline $\mathbf{1}$ & Frozen & +++ & + & + & +++ & ++ \\
& Paraffin & ++ & - & - & - & - \\
2 & Frozen & ++ & + & + & +++ & - \\
& Paraffin & ++ & - & - & - & - \\
3 & Frozen & ++ & - & + & ++ & ++ \\
& Paraffin & + & - & - & - & - \\
4 & Frozen & ++ & - & - & + & - \\
& Paraffin & + & - & - & - & - \\
5 & Frozen & ++ & - & - & - & - \\
& Paraffin & - & - & - & - & - \\
& Frozen & + & - & - & ++ & ++ \\
& Paraffin & + & - & - & - & - \\
\hline
\end{tabular}

fluorescence was less bright in the paraffin-embedded material. Of the two cases that showed positive staining for IgM on frozen section, only one was positive in the paraffin-embedded material. Of the two cases that were positive for $\operatorname{IgA}$ in frozen sections, neither was positive in paraffin sections. Of the two that were positive for fibrinogen, one was positive in the paraffin-embedded material. In addition, one case that stained for fibrinogen in the paraffin section had been negative in the frozen tissue.

\section{LUPUS ERYTHEMATOSUS}

Immunofluorescent staining for IgG, IgM, IgA, and fibrinogen at the dermal-epidermal junction was seen in formalin-fixed paraffin-embedded material. The pattern was thick and granular (Fig. 3), similar to that seen in frozen sections. The full results are shown in Table 3 . Of the six cases that showed positive staining for IgG and IgM in frozen sections, one was negative for IgG, and another was negative for IgM, using paraffin-embedded material. On staining for $\operatorname{IgA}$, five of the frozen sections were positive but only two of these were positive in the paraffin sections. Even though C3 was detected in all six cases in frozen section, all were negative in the paraffin-embedded material. Fibrinogen was demonstrated in the frozen sections in all six cases but only four cases were positive using paraffin sections.

\section{Discussion}

This study shows that the technique of direct immunofluorescence can be applied successfully to formalin-fixed paraffin-embedded skin biopsy specimens. Previous reports of immunofluorescence

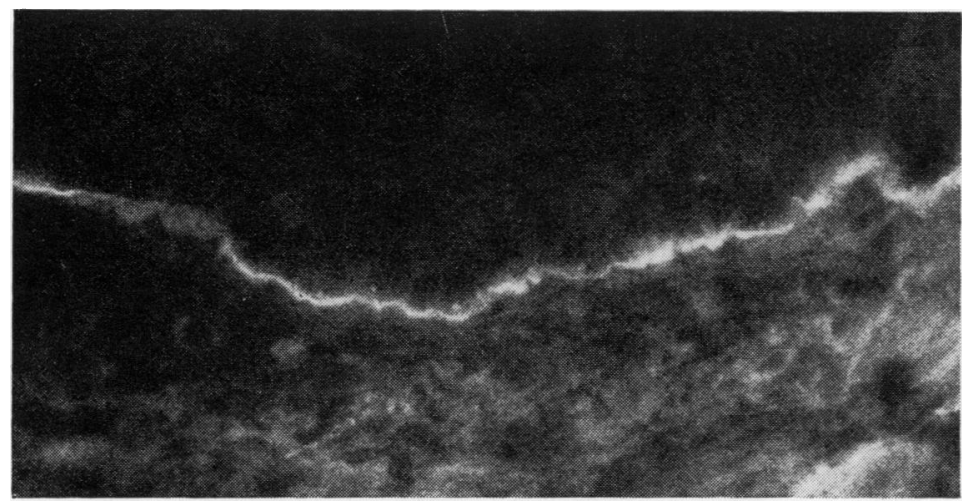

Fig. 2 Pemphigoid: basement membrane zone IgG. Paraffin section, direct immunofluorescence $\times 500$ 
Table 2 Pemphigoid: basement membrane zone staining

\begin{tabular}{lllllll}
\hline Case & Tissue & IgG & IgM & IgA & C3 & Fibrin \\
\hline 7 & Frozen & +++ & ++ & + & +++ & +++ \\
& Paraffin & ++ & - & - & ++ & + \\
8 & Frozen & ++ & - & ++ & ++ & - \\
& Paraffin & ++ & - & - & + & - \\
9 & Frozen & ++ & + & - & +++ & - \\
& Paraffin & + & - & - & ++ & ++ \\
10 & Frozen & ++ & - & - & + & +++ \\
& Paraffin & + & - & - & - & - \\
11 & Frozen & + & - & - & ++ & - \\
& Paraffin & + & - & - & ++ & - \\
12 & Frozen & + & - & - & - & - \\
& Paraffin & + & - & - & - & - \\
\hline
\end{tabular}

using paraffin-embedded material have included studies of lymphoid, thyroid, renal, pulmonary, and intestinal tissues, ${ }^{56}$ and the demonstration of intracellular antigens. ${ }^{67}$

In the present study dewaxed sections of the paraffin-embedded skin specimens were treated with trypsin as this is known to improve the detection of antigens. ${ }^{8} 9$ Even so, we have noted some loss of sensitivity when compared to the snap-frozen material in that the immunofluorescence in the paraffin-embedded sections was often diminished in intensity, and in some instances the antigens were undetectable. Attempts to improve the sensitivity by increasing the titre of antisera were unsuccessful. Some of the negative results, and the additional positive result for fibrinogen in the paraffin sections of case 9, may have been due to variation in the presence of antigens between different sites of biopsy; certainly deposits were sometimes present only in one area within any given biopsy site.

Of the antigens sought, $\mathrm{C} 3$ was the most difficult to demonstrate in formalin-fixed paraffin-embedded material. We were unable to detect $\mathrm{C} 3$ in any of the paraffin-embedded biopsy specimens of pemphigus or lupus erythematosus. Since then we have detected C3 in other cases of lupus erythematosus but not, so far, in paraffin-embedded material from pemphigus. In contrast, it proved easy to demonstrate $\mathbf{C} 3$ in cases of pemphigoid.

Taken together, these results show that the correct diagnosis would have been made in 17 out of the 18 cases examined if formalin-fixed paraffinembedded sections had been the only tissue available. Conversely, there would have been no false-positive diagnoses. Some artifactual epidermal fluorescence was occasionally encountered, but so far this has been easily recognised as such. In pemphigus, the immunofluorescence was entirely intercellular; in the upper epidermis it was characterised by a fine staining pattern between the cells, and in the mid epidermis it showed a spotted or speckled pattern (Fig. 1). In contrast, artifactual epidermal cell fluorescence was characterised by a patchy and denser fluorescence, which often involved the cells themselves, through all epidermal layers and where it was intercellular there was complete absence of any spotted appearance.

In many recent studies using formalin-fixed paraffin-embedded material, immunoperoxidase

Table 3 Lupus erythematosus: dermal-epidermal junction staining

\begin{tabular}{lllllll}
\hline Case & Tissue & IgG & IgM & IgA & C3 & Fibrin \\
\hline 13 & Frozen & ++ & +++ & ++ & -++ & +++ \\
& Paraffin & ++ & + & - & - & + \\
14 & Frozen & ++ & ++ & ++ & - & ++ \\
& Paraffin & ++ & ++ & - & - & ++ \\
15 & Frozen & ++ & + & +++ & - & + \\
& Paraffin & + & - & + & - & ++ \\
16 & Frozen & ++ & +++ & ++ & - & + \\
& Paraffin & - & + & - & - & - \\
17 & Frozen & + & + & - & - & +++ \\
& Paraffin & ++ & + & - & - & - \\
& Frozen & + & + & - & - & ++ \\
& Paraffin & + & + & - & - & + \\
\hline
\end{tabular}

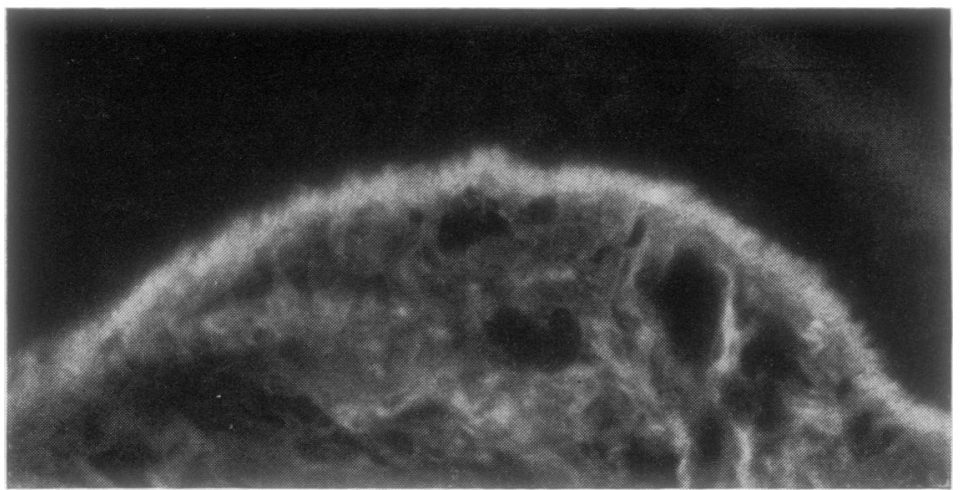

Fig. 3 Lupus erythematosus: dermal-epidermal juction IgG. Paraffin section, direct immunofluorescence $\times 500$ 
techniques have been used to detect immune deposits. ${ }^{1011}$ However, when compared with the immunofluorescence technique, immunoperoxidase techniques are not widely used and have proved more difficult to establish as a regular and reproducible diagnostıc investigation. It is for this reason that immunofluorescence was chosen for evaluation in this study.

In summary, the present results show that it is possible to use the technique of direct immunofluorescence to demonstrate immunoglobulins, complement, and fibrinogen in formalin-fixed paraffinembedded tissue sections of skin. Although the technique is less sensitive than when using frozen material the rate of diagnostic misinterpretation is low. Thus, when frozen tissue is not available, paraffin-embedded material can be used for direct immunofluorescence in the diagnosis of skin disease.

We are grateful to the Special Trustees of the Medical Research Committee, Bristol Health District (Teaching) for financial support. We also thank Drs Martlew and Ellis for ailowing us access to material from Princess Margaret Hospital, Swindon.

\section{References}

1 Jablonska S, Beutner EH, Michel B, et al. Uses for immunofluorescence tests of skin and sera; utilization of immunofluorescence in the diagnosis of bullous diseases, lupus erythematosus, and certain other dermatoses. Arch Dermatol 1975;111:371-81.

2 Tuffanelli DL. Cutaneous immunopathology: Recent observations. J Invest Dermatol 1975;65:143-53.

${ }^{3}$ Skeete MVH, Black MM. The evaluation of a special liquid fixative for direct immunofluorescence. Clin Exp Dermatol 1977;2:49-56.

${ }^{4}$ Beutner EH, Hale WL, Nisengard RJ, Chorzelski TP, Holubar K. In Immunopathology of the Skin: Labeled Antibody Studies. EH Beutner, TP Chorzelski, SF Bean, RE Jordan, eds. Stroudsburg, Pennsylvania: Dowden, Hutchinson, and Ross, 1973:213-7.

${ }^{5}$ Dorsett BH, Ioachim HL. A method for the use of immunofluorescence on paraffin-embedded tissues. Am J Clin Pathol 1978;69:66-72.

${ }^{6}$ Curran RC, Gregory J. Demonstration of immunoglobulin in cryostat and paraffin sections of human tonsil by immunofluorescence and immunoperoxidase techniques. J Clin Pathol 1978;31:974-83.

' Burns J, Hambridge M, Taylor CR. Intracellular immunoglobulins. A comparative study on three standard tissue processing methods using horseradish peroxidase and fluorochrome conjugates. J Clin Pathol 1974;27:548-57.

${ }^{8}$ Huang SN, Minassian H, More JD. Application of immunofluorescent staining on paraffin sections improved by trypsin digestion. Lab Invest 1976;35: 383-90.

${ }^{9}$ Curran RC, Gregory J. The unmasking of antigens in paraffin sections of tissue by trypsin. Experientia 1977;33:1400-1.

${ }^{10}$ Taylor CR, Burns J. The demonstration of plasma cells and other immunoglobulin-containing cells in formalin-fixed paraffin-embedded tissues using peroxidase-labelled antibody. J Clin Pathol 1973;27: 14-20.

${ }^{11}$ Burns J. Background staining and sensitivity of the unlabelled antibody-enzyme (PAP) method. Comparison with the peroxidase labelled antibody sandwich method using formalin fixed paraffin embedded material. Histochemistry 1975;43:291-4.

Requests for reprints to: Dr JWB Bradfield, Department of Pathology, Medical School, University Walk, Bristol BS8 1TD, UK. 